\title{
The effectiveness of battlefield acupuncture in addition to standard physical therapy treatment after shoulder surgery: a protocol for a randomized clinical trial
}

Michael S. Crowell ${ }^{1 *}$, Richard A. Brindle ${ }^{1}$, John S. Mason ${ }^{1}$, Will Pitt ${ }^{1}$, Erin M. Miller ${ }^{1}$, Matthew A. Posner ${ }^{2}$, Kenneth L. Cameron ${ }^{2}$ and Donald L. Goss ${ }^{1,3}$

\begin{abstract}
Introduction: There is a large incidence of shoulder instability among active young athletes and military personnel. Shoulder stabilization surgery is the commonly employed intervention for treating individuals with instability. Following surgery, a substantial proportion of individuals experience acute post-operative pain, which is usually managed with opioid pain medications. Unfortunately, the extended use of opioid medications can have adverse effects that impair function and reduce military operational readiness, but there are currently few alternatives. However, battlefield acupuncture (BFA) is a minimally invasive therapy demonstrating promise as a nonpharmaceutical intervention for managing acute post-operative pain.

Methods: This is a parallel, two-arm, single-blind randomized clinical trial. The two independent variables are intervention (2 levels, standard physical therapy and standard physical therapy plus battlefield acupuncture) and time (5 levels, 24 h, 48 h, 72 h, 1 week, and 4 weeks post shoulder stabilization surgery). The primary dependent variables are worst and average pain as measured on the visual analog scale. Secondary outcomes include medication usage, Profile of Mood States, and Global Rating of Change.

Discussion: The magnitude of the effect of BFA is uncertain; current studies report confidence intervals of between-group differences that include minimal clinically important differences between intervention and control groups. The results of this study may help determine if BFA is an effective adjunct to physical therapy in reducing pain and opioid usage in acute pain conditions.
\end{abstract}

Trial registration: ClinicalTrials.gov NCT04094246. Registered on 16 September 2019.

Keywords: Battlefield acupuncture, Opioids, Post-surgical pain

\footnotetext{
*Correspondence: michael_crowell@baylor.edu

'Baylor University - Keller Army Community Hospital Division 1 Sports

Physical Therapy Fellowship, West Point, NY, USA

Full list of author information is available at the end of the article
}

(c) The Author(s). 2020 Open Access This article is licensed under a Creative Commons Attribution 4.0 International License, which permits use, sharing, adaptation, distribution and reproduction in any medium or format, as long as you give appropriate credit to the original author(s) and the source, provide a link to the Creative Commons licence, and indicate if changes were made. The images or other third party material in this article are included in the article's Creative Commons licence, unless indicated otherwise in a credit line to the material. If material is not included in the article's Creative Commons licence and your intended use is not permitted by statutory regulation or exceeds the permitted use, you will need to obtain permission directly from the copyright holder. To view a copy of this licence, visit http://creativecommons.org/licenses/by/4.0/. The Creative Commons Public Domain Dedication waiver (http://creativecommons.org/publicdomain/zero/1.0/) applies to the data made available in this article, unless otherwise stated in a credit line to the data. 


\section{Background}

Shoulder and glenohumeral instability are serious problems for athletes and military personnel [1-6]. In general, younger and more active populations are at a high risk for glenohumeral instability [2]. However, military personnel sustain shoulder dislocations at a greater rate compared to the overall United States (U.S.) population (3.13 per 1000 person years) [2, 7, 8]. The risk of shoulder dislocation is greater still at Military Service Academies with an incidence of 4.35 per 1000 person years [5]. Given the highly physical demands of competitive athletics and military service, investigations of shoulder dislocation prevention and rehabilitation are warranted.

Management of shoulder dislocations usually includes shoulder stabilization surgery, which is considered the "gold standard" for treatment of shoulder dislocation in young, active patients [4,9-11]. This type of orthopedic surgery is associated with excellent short-term results and good long-term maintenance of shoulder function after chronic, unidirectional, and traumatic dislocation [4, 9-11]. However, as many as $80 \%$ of patients who undergo orthopedic surgery experience acute postoperative pain, which acutely impairs physical function and is a risk factor for the development of persistent pain [12]. Prolonged pain and physical impairment negatively affects military careers and impacts military readiness.

Physical therapy interventions focus on reducing pain and physical impairment associated with shoulder surgery. Standard care for patients post-shoulder surgery consists of two phases: (1) early rehabilitation from $24 \mathrm{~h}$ to 2 weeks post-surgery and (2) mid-term rehabilitation from 2 to 6 weeks post-surgery. Early rehabilitation after shoulder stabilization surgery focuses on pain control, protection of the surgical repair, prevention of a "stiff" shoulder, and regaining scapular control [13-15]. Within 6 weeks post-operative, the patient should achieve a minimum of $90^{\circ}$ of shoulder elevation and $10-20^{\circ}$ of external rotation at $50^{\circ}$ of scaption [13-15]. Elevated levels of pain may contribute to an inability to regain range of motion during early rehabilitation after shoulder stabilization surgery.

Opioid prescription is the widely employed method to manage post-operative pain in both Civilian and Military Health Systems. In representative civilian populations, $26 \%$ of patients undergoing arthroscopic shoulder procedures received at least one refill of their opioid prescription following surgery compared to $12 \%$ and $13 \%$ of patients following hip and knee procedures, respectively [16]. The Veteran's Affairs (VA) administration reported the average length of post-operative opioid use in all post-surgical patients as 15 days [17], which may be longer for shoulder post-surgical patients. Prolonged use of opioids post-surgery presents a possible risk for dependence and side effects such as drowsiness and impaired cognition, leading to necessary duty limitations that affect operational readiness. However, currently, there are few alternative pain management options. Thus, alternative and/or integrative methods of treating acute and chronic post-surgery pain without readiness reducing side-effects are needed.

Battlefield acupuncture (BFA), an auricular acupuncture protocol developed to treat acute and chronic pain in austere environments [18], may provide an integrative pain treatment option to decrease prescription medication usage for a myriad of musculoskeletal conditions [19-22]. The mechanisms of pain reduction by BFA are not fully understood. Chemical mechanisms for the treatment's effectiveness have been suggested and include endogenous opioid release and the inhibition of neurotransmitters [23, 24]. Multiple structures in the brain are responsible for processing and modulating painful stimuli to include the thalamus and sensory cortex. Limited research using functional magnetic resonance imaging and positive electron tomography has shown auricular acupuncture attenuates pain sensation within the sensory cortex, thereby reducing the perception of pain [25].

BFA has been taught and adopted throughout all branches of the Department of Defense (DoD) over the past two decades to provide alternatives to side effect laden prescription medications [22]. Despite wide-scale adoption by military medical providers, there is inconsistent evidence of BFA's effectiveness in reducing pain and opioid medication use. In a recent systematic review, pooled results indicated treatment of pain with auricular acupuncture had greater self-reported reductions in pain compared to sham interventions [21]. Studies of BFA in addition to standard care reported promising but inconsistent reductions in short-term pain. Patients with low back pain reported a greater reduction in pain associated with standard treatment supplemented with BFA compared to standard treatment alone [19]. However, following lower extremity surgery patients reported similar pain, opioid use, and quality of life with BFA plus standard care compared with standard of care alone or placebo treatments [26]. Most recently, BFA supplemented with standard care resulted in great pain reduction throughout the first week after shoulder surgery compared to standard care alone in a small cohort of U.S. Military Academy Cadets [27]. However, these findings were limited due to the small sample size and wide confidence intervals. More evidence is required to better understand the effectiveness of BFA to reduce pain in military populations.

BFA is an integrative pain treatment method that may be effective in reducing pain and opioid use postsurgery. The purpose of this study is to determine 
differences in pain, mood, self-reported improvement, and medication use during and after a standard physical therapy rehabilitation protocol supplemented with BFA, compared to a standard physical therapy rehabilitation protocol alone, for patients following shoulder stabilization surgery. The primary objective is to assess the effect of BFA on post-surgical pain (average and worst pain at $48 \mathrm{~h}$ and $72 \mathrm{~h}$ post-surgery). We hypothesize standard rehabilitation supplemented with BFA will produce greater reductions in pain compared to standard rehabilitation alone at $48 \mathrm{~h}$ and $72 \mathrm{~h}$ postsurgery.

Additional objectives are to assess the effect of BFA on: (1) medication use, (2) mood, and (3) self-reported improvement throughout the 4-week post-operative rehabilitation period, and (4) pain at 1 week and 4 weeks post-surgery. It is hypothesized that participants receiving standard rehabilitation supplemented with BFA will have greater reductions in medication use and improvements in patient's self-reported mood and improvement across the 4-week post-operative follow-up period. Further, participants receiving standard rehabilitation with BFA will have lower pain levels at 1 and 4 weeks postsurgery compared to those receiving standard rehabilitation only.

\section{Methods/design}

\section{Trial design}

This study is a parallel, two-arm single-blind randomized clinical trial. All participants will complete five study sessions following surgery: 24 h, 48 h, 72 h, 1 week, and 4 weeks post-surgery (Fig. 1). Participants will receive an email the day prior to each study session to promote retention and compliance. Data collection began in November 2019 and will continue for 4 years. All components of the study will be completed at the U.S. Military Academy at West Point, NY. The current Standard Protocol Items: Recommended for Interventional Trials (SPIRIT) guidelines for creating protocols for randomized clinical trials were followed (Supplemental Materials) [28]. Results of this trial will be reported in accordance with Consolidated Standards of Reporting Trials (CONSORT) Statement [29] and Template for Intervention Description and Replication (TIDieR) Checklist [30].

\section{Participants and study setting}

Participants will be recruited from the population of patients presenting to the Arvin Cadet Physical Therapy Clinic and the Keller Army Community Hospital (KACH) Physical Therapy and Orthopedic Clinics prior to and status-post shoulder stabilization surgery. A total of 105 male and female Department of Defense (DoD) beneficiaries, ages $17-55$ will be recruited for the study.
A 15\% drop-out rate is anticipated, which will result in at least 90 patients completing the study, with 45 patients per treatment group. On average $\mathrm{KACH}$ orthopedic surgeons perform eight shoulder stabilization surgeries monthly, suggesting the recruitment goal is feasible.

Inclusion criteria:

1. DoD beneficiaries age 18 to 55 years old (17 if cadet)

2. Prior to or within $24 \mathrm{~h}$ post shoulder stabilization surgery

3. Self-reported pain rating of at least 2 out of 10 on a Numerical Pain Rating Scale (NPRS)

Exclusion criteria:

1. Self-reported pregnancy

2. History of bloodborne pathogens, infectious disease, or active infection

3. History of metal allergy

4. History of bleeding disorders or currently taking anti-coagulant medications

5. Participants not fluent in English

\section{Randomization/allocation/blinding}

Participants will be screened prior to consent as part of routine clinical care by the investigative team during the first post-operative visit, $24 \mathrm{~h}$ after surgery. Those meeting criteria for inclusion will be informed as to the need and purpose of the research and invited to participate. Study participants will complete informed consent followed by a baseline examination (Supplemental Materials). Consenting participants will be randomized into one of two groups via a concealed allocation process: the control group (standard physical therapy rehabilitation) or the intervention group (BFA plus a standard physical therapy rehabilitation). Following informed consent and baseline examination, a second investigator blinded to the baseline examination will open a sealed envelope containing a folded index card labeled with the participant's group assignment. An investigator not involved with participant recruitment or data collection will create the randomization sequence with a 1:1 allocation using a random permuted block approach on Excel 2010 (Microsoft, Redmond, WA, USA) [31]. The random permuted block approach was utilized to keep intervention arms relatively equivalent throughout the data collection process. Group assignment will be recorded with a unique participant identifier and secured in a separate folder until completion of all data collection through the final follow-up.

Participants and the treating physical therapists will not be blinded to group assignment. Verification of the medication $\log$ by the outcome assessor may be another source of bias. 


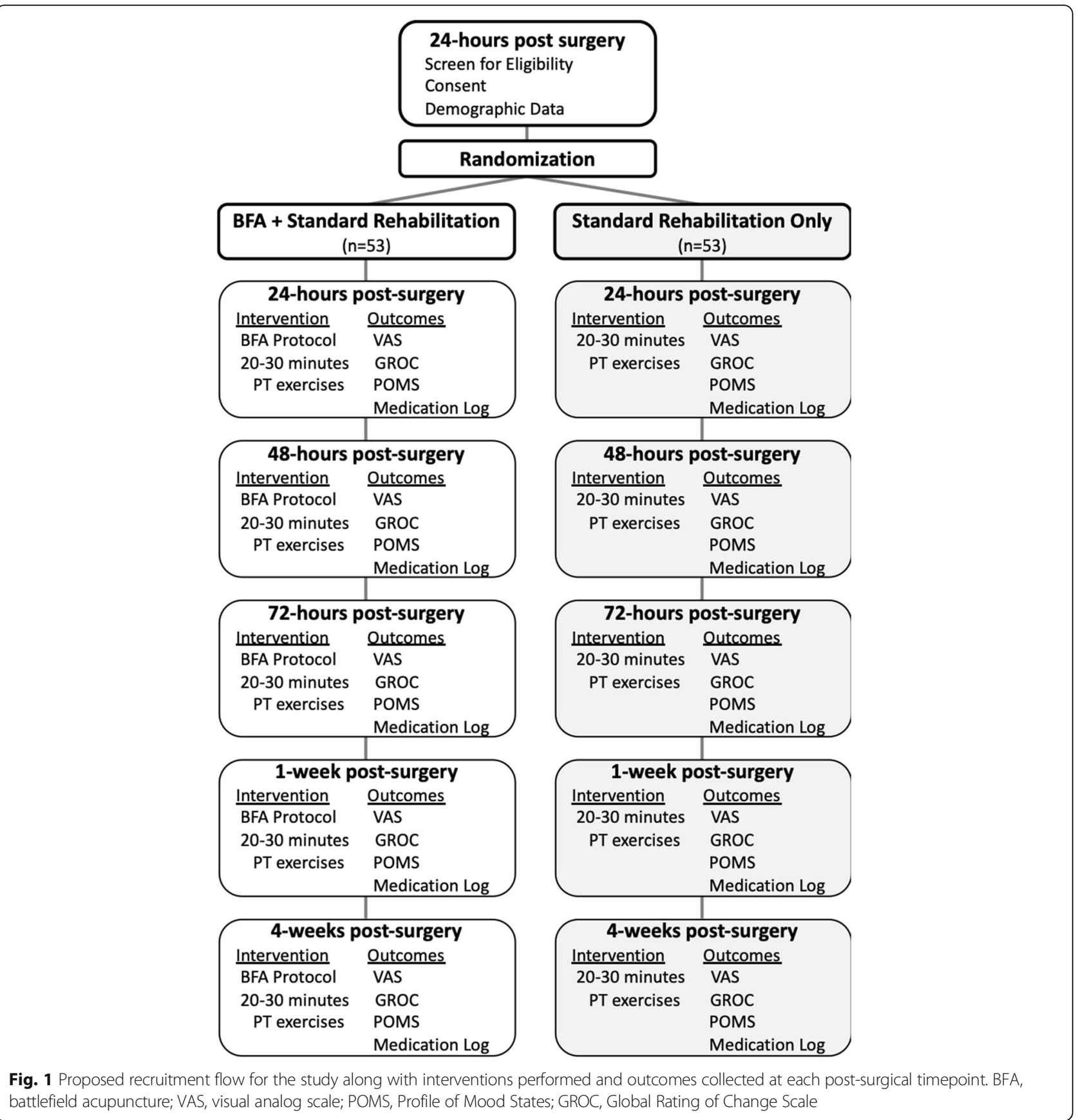

Outcome assessors who record pain, self-report function, and mood surveys, perform data reduction, and perform data analysis will be blinded to the participants' treatment group. Participants frequently require clarification of outcome measure instructions during completion of data collection forms and may require additional assistance if the surgery is performed on their dominant extremity. Participants will interact with the outcome assessor and complete data collection forms behind a closed curtain, where the outcome assessor is not able to see the patient. Non-standard blinding techniques are often implemented in trials assessing nonpharmacological treatments and may minimize either conscious or unconscious researcher recording and reporting bias during completion and verification of the data collection forms [32].

\section{Interventions}

\section{Standard physical therapy rehabilitation (active control} group)

Both groups will receive standard post-surgical physical therapy according to guidelines developed at our institution (Additional file 2). Five physical therapy sessions, 
approximately $30 \mathrm{~min}$ in duration, will occur at the 24-h initial time point and at the 48-h, 72-h, 1-week, and 4week follow-up visits. During each visit, the physical therapist will expose the surgical site to check for any signs of infection and review the post-operative precautions with the patient. The patient will perform range of motion and muscle activation exercises consisting of modified pendulum exercises, active range of motion exercises for the elbow/wrist/hand, active-assisted shoulder flexion and external rotation, gentle isometric muscle activation of the rotator cuff and deltoid, and scapular muscle activation exercises. Ice and intermittent compression will be applied for control of pain and swelling, as needed. Patients utilizing these rehabilitation guidelines after shoulder stabilization surgery have demonstrated significant improvements in pain, range of motion, and function that meet established goals for progression to the next phase of rehabilitation [33].

\section{Battlefield acupuncture (study intervention group)}

Using aseptic technique (proper handwashing, personal protective equipment (PPE), and ear cleansing with an alcohol swab), auricular acupuncture using the five points specified within the BFA protocol will be administered. Each ear will potentially be punctured with ASP needles at five sequential points: cingulate gyrus, thalamus, omega 2, point zero, and Shen-Men (Fig. 2, Additional file 3). The acupuncture sequence will begin on the same side of the shoulder surgery (ipsilateral ear) and begin at the cingulate gyrus point. Following each ASP needle placement, the participant will be asked to stand and move/walk for at least $30 \mathrm{~s}$ while being monitored for any side effects, including light-headedness, dizziness/loss of balance or nausea. Additionally, selfreported current pain level will be reassessed. If the participant's pain is above zero to one out of 10 on the NPRS, the contralateral ear will be punctured with the ASP needle in the cingulate gyrus. ASP needle application will continue, alternating between ipsilateral and contralateral ears in order through the remaining four points, until the desired pain level of zero to one out of 10 is achieved or until all 10 ASP needles are placed.

There is no standard time for the ASP needles to remain in the participant's ears, but they may remain up to 3-5 days as they naturally work their way out of the skin. Participants will be instructed on how to properly

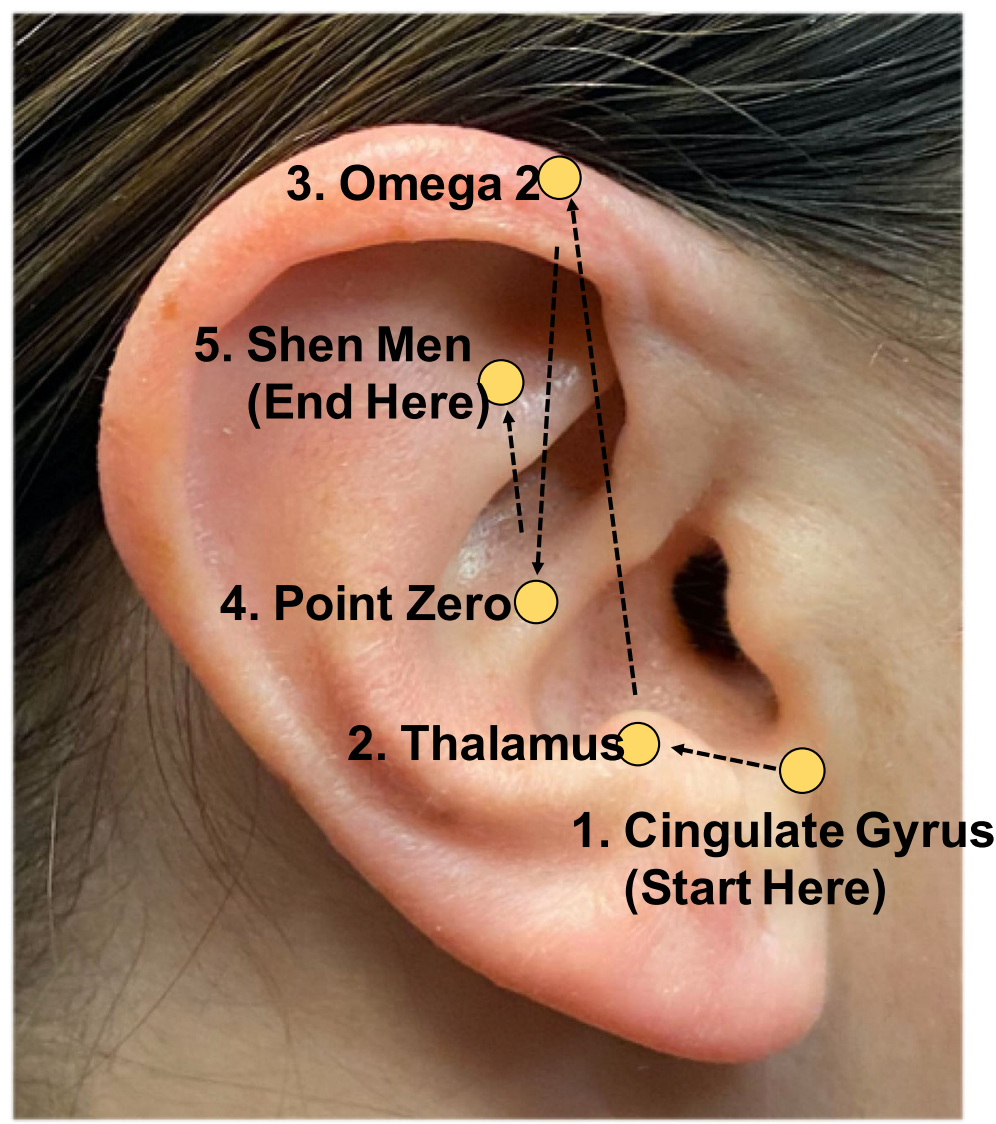

Fig. 2 The five auricular acupuncture points of the battlefield acupuncture protocol 
care for and remove the ASP needles should they become irritating. There are no documented cases of loss of treatment effect should participants remove ASP needles prematurely to their natural falling out. All participants in the study intervention group will receive BFA treatment at the initial (24-h) time point. Repeat treatment intervention may be provided during the 48-h, 72$\mathrm{h}$, and 1-week follow-up visits, but will not coincide with standard physical therapy rehabilitation sessions. The decision whether BFA intervention will be provided during the follow-up visits of the intervention group participants will be left to the discretion of the treating physical therapist and preference of the patient. Treating physical therapists will take into account the patient's current pain level and prior response to BFA. Additionally, for each participant, the number of BFA interventions executed, the number of ASP needles inserted at each intervention, and the location of each needle's placement will be recorded for descriptive analyses. If ASP needles remain in place at follow-up visits and the patient requests additional treatment, additional ASP needles will be placed adjacent to locations used previously.

All BFA treatments will be performed by investigators trained and certified on the standard BFA protocol developed by Dr. Niemtzow [18]. Participants in the intervention group will continue to receive the standard of care in accordance with the post-operative protocol between study follow-up visits.

\section{Outcome measures}

Demographic characteristics will be recorded and include sex, age, ethnicity, military demographics, height, weight, and surgical history. The primary outcome measure will be pain (average and worst pain over the past $24 \mathrm{~h}$, assessed on the visual analog scales [VAS]) at $24 \mathrm{~h}, 48 \mathrm{~h}, 72 \mathrm{~h}, 1$ week, and 4 weeks after surgery. Two previous studies suggest that the greatest effect of BFA on pain occurs at 1 week or less [27, 34]. Secondary outcome measures will be medication use (opioids and nonopioid medications), patient self-reported mood (Profile of Mood States [POMS]), and patient self-reported improvement (Global Rating of Change Scale [GROC]). All participants will complete all outcome measures at five post-surgical timepoints: $24 \mathrm{~h}, 48 \mathrm{~h}, 72 \mathrm{~h}, 1$ week, and 4 weeks (Fig. 1).

The VAS assesses the perception of pain intensity by asking the patient to mark their level of pain along a $100-\mathrm{mm}$ line, where the left limit indicates no pain and the right limit indicates the worst pain imaginable [35, 36]. The VAS is a valid and reliable measure of pain intensity [35-39] with a minimal clinically important difference (MCID) of $10 \mathrm{~mm}$ and patient acceptable symptoms state of $30 \mathrm{~mm}$ in acute, post-operative pain [38].

The POMS is a 40-item questionnaire designed to measure the transient emotional states of tensionanxiety, depression-dejection, fatigue-inertia, vigoractivity, confusion-bewilderment, and anger-hostility in sports and other settings [40]. The POMS is a valid and reliable measure of mood in athletes and sports [40, 41].

The GROC is a 15 -point self-report Likert scale $(-7$ to +7 , with -7 equaling a very great deal worse, zero equaling no change or improvement, and +7 equaling a very great deal better) of patient-perceived status since the onset of treatment [42]. The GROC is valid and reliable with an MCID of two points [42], although numerous studies define major improvement as five or greater.

At each visit, the physical therapist will assess the surgical site and neurological status of the patient. The area of treatment will be examined for those patients who have received BFA. Any side effects or adverse events will be recorded in the patient's electronic medical record and either treated or referred to an appropriate medical provider for treatment. Serious adverse events will be reported to the IRB in accordance with the approved study protocol. At the conclusion of the study, each patient's medical record will be screened for any adverse events.

\section{Data analysis}

An a priori power analysis was performed using G*Power, version 3.1.9.2 (Heinrich-Heine-Universitat Dusseldorf, Dusseldorf, Germany) with $\alpha=0.05, \beta=0.80$, and an effect size of 0.6 (change in VAS worst pain between $24 \mathrm{~h}$ and 1 week post-surgery), resulting in a required sample of 90 participants. The effect size was determined through examination of data from a previous published study at the same institution [27]. To account for a potential drop-out rate of $15 \%$, a total of 105 participants will be enrolled.

Descriptive statistics, including measures of central tendency and dispersion, will be calculated for demographic data. Frequency distributions will be estimated for categorical data. Four separate 2-by-5 mixed-model analyses of variance (ANOVA) with group as the between-subjects factor (BFA plus standard physical therapy rehabilitation versus standard physical therapy rehabilitation alone) and time as the repeated measure within-subjects factor ( $24 \mathrm{~h}, 48 \mathrm{~h}, 72 \mathrm{~h}, 1$ week, 4 weeks) to determine the effect of BFA on pain, medication use, self-reported improvement (GROC), and mood (POMS) over the 4-week post-operative period. Alpha will be set at 0.05 for all omnibus comparisons, which are the group"time interaction, the main effect for group (fixed factor), and the main effect for time (repeated measure). Planned pairwise comparisons will be performed to 
examine significant main effects for group using independent $t$ test, and time using paired $t$ tests. Alpha for planned pairwise comparisons will be corrected using the Sidak's correction to control for family-wise type I error. The Cohen $d$ coefficient will be used to assess the effect size between pairwise comparisons. Prior to performance of the ANOVAs, all outcome measures will be assessed for normality. The appropriate non-parametric statistical tests will be used for any non-normality distributed outcomes.

When post-intervention data points are missing, data will be replaced using multiple imputation for participants who received their allocated intervention [43]. All statistical analyses will be performed with the statistical package SPSS version 26 (IBM, Chicago, IL, USA).

\section{Discussion}

The purpose of this study is to determine differences in pain, mood, self-reported improvement, and medication use during and after a standard physical therapy rehabilitation protocol supplemented with BFA, compared to a standard physical therapy rehabilitation protocol alone, for patients following shoulder stabilization surgery. BFA may be an effective adjunct to physical therapy to reduce pain and opioid utilization in patients with acute pain $[21,27,34]$. However, the magnitude of the effect of BFA is uncertain and current studies lack blinding of outcomes assessors [21, 27, 34]. The results of this study may help determine if BFA is an effective adjunct to standard physical therapy rehabilitation post-shoulder surgery in reducing pain and opioid usage. Specifically, this study will determine if pain intensity differs between standard rehabilitation supplemented with BFA and standard rehabilitation alone during the short-term at $48 \mathrm{~h}$ and $72 \mathrm{~h}$ post-surgery, and longer-term at 1 week and 4 weeks post-surgery. Additionally, this study will determine if participants' medication use and selfreported mood and perceptions of improvement in function differ between standard rehabilitation with and without supplemental BFA across the 4-week postoperative follow-up period.

This study is not without limitations and design constraints. The primary limitation is physical therapists and patients are not blinded to treatment groups. Many non-physiologic factors, including placebo effects, may contribute to treatment response in patients who have received BFA. We elected to forgo a sham treatment group due to the lack of feasibility in maintaining a realistic sham treatment at our institution. The most recent study of BFA that utilized a sham intervention applied the treatment while the patient was still under anesthesia (Crawford 2019). BFA represents a low-risk and lowcost alternative method of pain control when compared to opioid medications, making the potential for a placebo contribution to the response to treatment more acceptable [44, 45]. Blinding of outcomes assessors to group allocation will be conducted to minimize bias associated.

\section{Trial status}

This study was approved by the RHC-A IRB; protocol ID number 19KACH0003, initially approved 3 September 2019, modification approved 28 October 2019. Recruitment began 25 September 2019 and will be tentatively completed in December 2022.

\section{Supplementary Information}

Supplementary information accompanies this paper at https://doi.org/10. 1186/s13063-020-04909-8.

Additional file 1.SPIRIT 2013 Checklist

Additional file 2. Shoulder Stabilization Rehabilitation Guidelines

Additional file 3. Battlefield Acupuncture Intervention

\section{Abbreviations}

ANOVA: Analysis of variance; ASP: Aiguille semi-permanente; BFA: Battlefield acupuncture; CONSORT: Consolidated Standards of Reporting Trials; DoD: Department of Defense; GROC: Global Rating of Change Scale; KACH: Keller Army Community Hospital; MCID: Minimal clinically important difference; POMS: Profile of Mood States; SPIRIT: Standard Protocol Items: Recommended for Interventional Trials; TIDieR: Template for Intervention Description and Replication; VA: Veteran's administrations; VAS: Visual analogue scale

\section{Acknowledgements}

This work was supported in part by the Uniformed Services University, Department of Physical Medicine \& Rehabilitation, Musculoskeletal Injury Rehabilitation Research for Operational Readiness (MIRROR)

(HU00011920011). The authors would like to specifically thank Ms. Linzie Wagner, Ms. Whitley Lucio, and Mr. Brock Heller for their hard work and expertise in the management, regulatory guidance, and data analytics of the project.

\section{Authors' contributions}

Author roles and responsibilities. PI, Principal Investigator; Al, Assistant Investigator; BFA, battlefield acupuncture. Study personnel: MSC; Study role: Pl; Responsibilities: BFA treatments per study protocol, participant recruitment/consent, protocol development, manuscript preparation. Study personnel: RAB; Study role: Al; Responsibilities: Project management, participant recruitment/consent, protocol development, blinded outcomes assessor, data analysis, manuscript preparation. Study personnel: JSM; Study role: Al; Responsibilities: BFA treatments per study protocol, participant recruitment/consent, protocol development, manuscript preparation. Study personnel: WJP; Study role: Al; Responsibilities: Protocol development, blinded outcomes assessor, data analysis, manuscript preparation. Study personnel: EMM; Study role: Al; Responsibilities: Protocol development, blinded outcomes assessor, manuscript preparation. Study personnel: MAP; Study role: Al; Responsibilities: Participant recruitment, surgeon consultant. Study personnel: KLC; Study role: Al; Responsibilities: Participant recruitment, statistical consultant. Study personnel: DLG; Study role: Al; Responsibilities: Protocol development, manuscript preparation. Author contributions are consistent with the Contributor Roles Taxonomy (CRediT) (docs.casrai.org/ CRediT) methodology for attributing contributions. The authors read and approved the final manuscript.

\section{Funding}

This work was supported by a grant from the Musculoskeletal Injury Rehabilitation Research for Operational Readiness (MIRROR) program at the Uniformed Services University of the Health Sciences (USUHS). USUHS/ 
MIRROR did/will not have a role in the study design; collection, analysis, interpretation of data, and writing of the report. USUHS/MIRROR provided technical assistance with regulatory approval and data management systems. USUHS/MIRROR will have to approve the content of the final report submission.

\section{Availability of data and materials}

The coded electronic research data for this study will be stored in Research Electronic Data Capture (REDCap), an encrypted, access-controlled, passwordprotected electronic data capture and management system housed on a DoD server and maintained by the Uniformed Services University of the Health Sciences Information Technology. Data from the study are available by email request to the lead author for the purpose of systematic review and meta-analysis.

\section{Ethics approval and consent to participate}

An ethics review was conducted by the Keller Army Community Hospital (KACH) Human Research Protections Office and will be monitored by the Regional Health Command - Atlantic (RHC-A) Institutional Review Board (IRB) to ensure compliance with federal regulations for the protection of human medical research subjects. This study was approved by the RHC-A IRB; protocol ID number 19KACH0003. Written informed consent will be obtained from all participants. Any adverse events resulting from participation in the study, or compromise of data security will be immediately reported by the Primary Investigator to the IRB according to established policies and procedures.

\section{Consent for publication}

Informed consent was obtained for publication of images depicted in Fig. 2 and Additional file 3 of this manuscript.

\section{Competing interests}

The authors completed the International Committee of Medical Journal Editors (ICJME) form for disclosure of potential conflicts of interest. They reported no conflicts of interest.

\section{Author details}

${ }^{1}$ Baylor University - Keller Army Community Hospital Division 1 Sports Physical Therapy Fellowship, West Point, NY, USA. ${ }^{2}$ John A Feagin, Jr. Sports Medicine Fellowship, Keller Army Community Hospital, West Point, NY, USA. ${ }^{3}$ Department of Physical Therapy, High Point University, High Point, NC, USA.

Received: 29 April 2020 Accepted: 16 November 2020

Published online: 03 December 2020

\section{References}

1. Amako M, Arino H, Tsuda Y, Tsuchihara T, Nemoto K. Recovery of shoulder rotational muscle strength after arthroscopic bankart repair. Orthop J Sports Med. 2017;5:2325967117728684. https://doi.org/10.1177/2325967117728684 journals.sagepub.com

2. Owens BD, Dawson L, Burks R, Cameron KL. Incidence of shoulder dislocation in the United States military: demographic considerations from a high-risk population. J Bone Joint Surg - Ser A. 2009;91:791-6. https://doi. org/10.2106/JBJS.H.00514.

3. Brelin A, Dickens J. Posterior shoulder instability. Sports Med Arthrosc Rev. 2017;25:136-43.

4. Owens BD, Agel J, Mountcastle SB, Cameron KL, Nelson BJ. Incidence of glenohumeral instability in collegiate athletics. Am J Sports Med. 2009;37: 1750-4.

5. Owens BD, Duffey ML, Nelson BJ, DeBerardino TM, Taylor DC, Mountcastle SB. The incidence and characteristics of shoulder instability at the United States Military Academy. Am J Sports Med. 2007;35:1168-73.

6. Waterman B, Owens BD, Tokish JM. Anterior shoulder instability in the military athlete. Sports Health. 2016;8:514-9.

7. Kardouni JR, Mckinnon CJ, Seitz AL. Incidence of shoulder dislocations and the rate of recurrent instability in soldiers. Med Sci Sports Exerc. 2016;48: 2150-6. https://doi.org/10.1249/MSS.0000000000001011.

8. Cameron KL, Mauntel TC, Owens BD. The epidemiology of glenohumeral joint instability: incidence, burden, and long-term consequences. Sports Med Arthrosc Rev. 2017;25:144-9. https://doi.org/10.1097/JSA. 0000000000000155 .
9. Longo UG, Loppini M, Rizzello G, Ciuffreda M, Maffulli N, Denaro V. Management of primary acute anterior shoulder dislocation: systematic review and quantitative synthesis of the literature. Arthroscopy. 2014;30: $506-22$.

10. Bottoni CR, Wilckens JH, DeBerardino TM, D'Alleyrand JCG, Rooney RC, Harpstrite JK, et al. A prospective, randomized evaluation of arthroscopic stabilization versus nonoperative treatment in patients with acute, traumatic, first-time shoulder dislocations. Am J Sports Med. 2002;30:576-80.

11. Godin J, Sekiya JK. Systematic review of rehabilitation versus operative stabilization for the treatment of first-time anterior shoulder dislocations. Sports Health. 2010;2:156-65.

12. Veal FC, Bereznicki LRE, Thompson AJ, Peterson GM, Orlikowski C. Subacute pain as a predictor of long-term pain following orthopedic surgery: an Australian prospective 12 month observational cohort study. Medicine. 2015;94:e1498.

13. Johnson M. Rehabilitation following surgery for Glenohumeral instability. Sports Med Arthrosc. 2017;25:116-22.

14. DeFroda SF, Mehta N, Owens BD. Physical therapy protocols for arthroscopic bankart repair. Sports Health. 2018;10:250-8.

15. Gaunt BW, Shaffer MA, Sauers EL, Michener LA, Mccluskey GM, Thigpen CA. The American Society of Shoulder and Elbow Therapists' consensus rehabilitation guideline for arthroscopic anterior capsulolabral repair of the shoulder. J Orthop Sports Phys Ther. 2010;40:155-68.

16. Sheth U, Mehta M, Huyke F, Terry MA, Tjong VK. Opioid use after common sports medicine procedures: a systematic review. Sports Health. 2020;12: 225-33. https://doi.org/10.1177/1941738120913293.

17. Mudumbai SC, Oliva EM, Lewis ET, Trafton J, Posner D, Mariano ER, et al. Time-to-cessation of postoperative opioids: a population-level analysis of the Veterans Affairs Health Care System. Pain Med. 2016;17:1732-43. https:// doi.org/10.1093/pm/pnw015.

18. Niemtzow RC. Battlefield acupuncture. Med Acupunct. 2007;19:225-8. https://doi.org/10.1089/acu.2007.0603.

19. Usichenko TI, Hermsen M, Witstruck T, Hofer A, Pavlovic D, Lehmann C, et al. Auricular acupuncture for pain relief after ambulatory knee arthroscopy-a pilot study. Evid Based Complement Alternat Med. 2005;2: 185-9. https://doi.org/10.1093/ecam/neh097.

20. Moss DA, Crawford P. Ear acupuncture for acute sore throat: a randomized controlled trial. J Am Board Fam Med. 2015;28:697-705. https://doi.org/10. 3122/jabfm.2015.06.150014.

21. Jan AL, Aldridge ES, Rogers IR, Visser EJ, Bulsara MK, Niemtzow RC. Does ear acupuncture have a role for pain relief in the emergency setting? A systematic review and meta-analysis. Med Acupunct. 2017;29:276-89. https://doi.org/10.1089/acu.2017.1237.

22. Walker PH, Pock A, Ling CG, Kwon KN, Vaughan M. Battlefield acupuncture: opening the door for acupuncture in Department of Defense/Veteran's Administration health care. Nurs Outlook. 2016;64:491-8.

23. Soliman N, Frank BL. Auricular acupuncture and auricular medicine. Phys Med Rehabil Clin N Am. 1999;10:547-54.

24. Clement-Jones V, McLoughlin L, Tomlin S, Besser GM, Rees LH, Wen HL. Increased $\beta$-endorphin but not Met-enkephalin levels in human cerebrospinal fluid after acupuncture for recurrent pain. Lancet. 1980;316:946-.

25. Cho ZH, Oleson TD, Alimi D, Niemtzow RC. Acupuncture: the search for biologic evidence with functional magnetic resonance imaging and positron emission tomography techniques. J Altern Complement Med. 2002;8:399-401. https://doi.org/10.1089/107555302760253577.

26. Crawford P, Moss DA, Crawford AJ, Sharon DJ. Modified battlefield acupuncture does not reduce pain or improve quality of life in patients with lower extremity surgery. Mil Med. 2019;184(Suppl 1):545-9. https://doi. org/10.1093/milmed/usy277.

27. Collinsworth KM, Goss DL. Battlefield acupuncture and physical therapy versus physical therapy alone after shoulder surgery. Med Acupunct. 2019; 31:228-38. https://doi.org/10.1089/acu.2019.1372.

28. Chan AW, Tetzlaff JM, Altman DG, Laupacis A, Gøtzsche PC, Krleža-Jerić K, et al. SPIRIT 2013 statement: defining standard protocol items for clinical trials. Ann Intern Med. 2013;158:200-7. https://doi.org/10.7326/0003-4819158-3-201302050-00583.

29. Schulz KF, Altman DG, Moher D, Group C. CONSORT 2010 statement: updated guidelines for reporting parallel group randomised trials. Trials. 2010;11:32. https://doi.org/10.1186/1745-6215-11-32.

30. Hoffmann TC, Glasziou PP, Boutron I, Milne R, Perera R, Moher D, et al. Better reporting of interventions: template for intervention description and replication (TIDieR) checklist and guide. BMJ. 2014;348:g1687. 
31. Altman DG, Bland JM. How to randomise. BMJ. 1999;319:703-4. https://doi. org/10.1136/bmj.319.7211.703.

32. Boutron I, Guittet L, Estellat C, Moher D, Hróbjartsson A, Ravaud P. Reporting methods of blinding in randomized trials assessing nonpharmacological treatments. PLoS Med. 2007;4:0370-80.

33. Halle R, Crowell M, Goss D. Dry needling and physical therapy versus physical therapy alone following shoulder stabilization repair: a randomized clinical trial. Int J Sports Phys Ther. 2020;15:81-102.

34. Fox LM, Murakami M, Danesh $H$, Manini AF. Battlefield acupuncture to treat low back pain in the emergency department. Am J Emerg Med. 2018;36: 1045-8. https://doi.org/10.1016/j.ajem.2018.02.038.

35. Price DD, Bush FM, Long S, Harkins SW. A comparison of pain measurement characteristics of mechanical visual analogue and simple numerical rating scales. Pain. 1994;56:217-26. https://doi.org/10.1016/0304-3959(94)90097-3.

36. Price DD, McGrath PA, Rafii A, Buckingham B. The validation of visual analogue scales as ratio scale measures for chronic and experimental pain. Pain. 1983;17:45-56. https://doi.org/10.1016/0304-3959(83)90126-4.

37. Lee JS, Hobden E, Stiell IG, Wells GA. Clinically important change in the visual analog scale after adequate pain control. Acad Emerg Med. 2003;10: 1128-30.

38. Myles PS, Myles DB, Galagher W, Boyd D, Chew C, MacDonald N, et al. Measuring acute postoperative pain using the visual analog scale: the minimal clinically important difference and patient acceptable symptom state. Br J Anaesth. 2017;118:424-9. https://doi.org/10.1093/bja/aew466.

39. Briggs M. A descriptive study of the use of visual analogue scales and verbal rating scales for the assessment of postoperative pain in orthopedic patients. J Pain Symptom Manag. 1999;18:438-46.

40. Grove JR, Prapavessis H. Preliminary evidence for the reliability and validity of an abbreviated Profile of Mood States. Int J Sport Psychol. 1992;23:93-109.

41. Leunes A, Burger J. Profile of Mood States research in sport and exercise psychology: past, present, and future. J Appl Sport Psychol. 2000;12:5-15

42. Kamper S. Global Rating of Change Scales: a review of strengths and weaknesses and considerations for design. J Man Manip Ther. 2009;17:163-70.

43. Sterne JAC, White IR, Carlin JB, Spratt M, Royston P, Kenward MG, et al. Multiple imputation for missing data in epidemiological and clinical research: potential and pitfalls. BMJ. 2009;339:157-60.

44. Bialosky JE, Bishop MD, Penza CW. Placebo mechanisms of manual therapy: a sheep in Wolf's clothing? J Orthop Sports Phys Ther. 2017;47:301-4.

45. Benz LN, Flynn TW. Placebo, nocebo, and expectations: leveraging positive outcomes. J Orthop Sports Phys Ther. 2013;43:439-41.

\section{Publisher's Note}

Springer Nature remains neutral with regard to jurisdictional claims in published maps and institutional affiliations.

Ready to submit your research? Choose BMC and benefit from:

- fast, convenient online submission

- thorough peer review by experienced researchers in your field

- rapid publication on acceptance

- support for research data, including large and complex data types

- gold Open Access which fosters wider collaboration and increased citations

- maximum visibility for your research: over $100 \mathrm{M}$ website views per year

At $\mathrm{BMC}$, research is always in progress.

Learn more biomedcentral.com/submissions 